\title{
Unified equations of state for cold nonaccreting neutron stars with Brussels-Montreal functionals. III. Inclusion of microscopic corrections to pasta phases
}

\author{
J. M. Pearson ${ }^{1}$ and N. Chamel $\odot^{2}$ \\ ${ }^{1}$ Département de Physique, Université de Montréal, Montréal (Québec), H3C 3J7, Canada \\ ${ }^{2}$ Institut d'Astronomie et d'Astrophysique, CP-226, Université Libre de Bruxelles, 1050 Brussels, Belgium
}

(Received 14 July 2021; accepted 15 December 2021; published 5 January 2022)

\begin{abstract}
Earlier fourth-order extended Thomas-Fermi calculations with functional BSk24 of pasta phases in the inner crust of neutron stars are here repeated with Strutinsky-integral and pairing corrections included for protons. The main consequence of these corrections is to eliminate the spaghetti phase at zero temperature and significantly reduce the range of densities over which the lasagna phase is found. On the other hand, the microscopic corrections have a negligible impact on the equation of state and on the proton fraction.
\end{abstract}

DOI: $10.1103 /$ PhysRevC.105.015803

\section{INTRODUCTION}

Three distinct regions can be recognized in neutron stars (see, e.g., Ref. [1] for a recent review). The outermost of these regions, the "outer crust," consists of an assembly of bound nuclei and electrons that globally is electrically neutral. The. nuclei in this region become more and more neutron rich with increasing depth, until at a mean baryon number density $\bar{n}$ of around $2.6 \times 10^{-4} \mathrm{fm}^{-3}$ unbound neutrons appear. This "neutron drip" marks the transition to the "inner crust," an inhomogeneous assembly of neutron-proton clusters and unbound neutrons, neutralized by electrons. By the point where $\bar{n}$ has risen to about $0.08 \mathrm{fm}^{-3}$ the inhomogeneities have been smoothed out: The "core" of the star has been reached.

In 2018 we published unified calculations of the equation of state (EoS) and the composition of all three regions, the same highly realistic nuclear energy-density functional being used throughout [2]. [A unified treatment of all three regions was previously carried out in Ref. [3], adopting the Thomas-Fermi (TF) approximation for the inner crust.] In our 2018 paper the inner crust was calculated in the framework of spherical Wigner-Seitz (WS) cells using the fourth-order extended Thomas-Fermi plus Strutinsky integral (ETFSI) method [4-7]. This is a high-speed approximation to the Hartree-Fock-Bogoliubov (HFB) method (for a review see, e.g., Ref. [8]), consisting of two distinct stages: a full ETF treatment of the kinetic-energy and spin-current densities, followed by the addition of shell corrections, calculated by the Strutinsky-integral method (SI), and pairing corrections, handled in the Bardeen-Cooper-Schrieffer (BCS) approximation (see, e.g., Ref. [9] for recent comparisons between the HFB and ETFSI methods in neutron-star crusts). Only the proton corrections were taken into account; our neglect of neutron shell corrections in the inner crust is discussed in the next paragraph.

More recently [10] we extended our earlier work [2] on the functional BSk24 [11] to allow the WS cells of the inner crust to assume nonspherical shapes. Specifically, we considered, as an alternative to spherical shapes, infinitely long cylinders ("spaghetti") and plates of infinite extent ("lasagna"). These "pasta" calculations [10], unlike our calculations on spherical WS cells [2], were pure ETF, the neglect of shell and pairing corrections for the pasta phases being justified at the time with the argument that they should be negligible for pasta. In this paper we investigate this issue more closely and repeat the pasta calculations of Ref. [10] with the SI and pairing corrections taken into account for protons. As in the sphericalcell calculations of Ref. [2], we will neglect these corrections for the neutrons. In the past we justified this neglect on the grounds that the spectrum of unbound occupied singleparticle (s.p.) neutron states was continuous, with the result that any neutron added to the system must go into this continuum. It follows that we should not expect any of the fluctuations characteristic of shell effects [5]. We will revisit the question of neutron shell corrections at the end of this section.

To summarize some essential points of Refs. [2,10], we write the total energy per nucleon as

$$
e=e_{\mathrm{Sky}}+e_{C}+e_{e}-Y_{p} Q_{n, \beta} .
$$

Here the first term denotes the total nuclear energy per nucleon corresponding to our chosen generalized Skyrme functional, the second and third terms the Coulomb and electronic kinetic energy per nucleon, respectively, while the last term takes account of the neutron-proton mass difference, $Q_{n, \beta}$ $(=0.782 \mathrm{MeV})$ being the $\beta$-decay energy of the neutron (see Eq. (13) of Ref. [2]). In this last term $Y_{p}$ is the proton fraction $Z / A, Z$ and $A$ being respectively the numbers of protons and nucleons in the WS cell for the spherical case, the numbers per unit length of cylindrical cells or the numbers per unit area of platelike cells. The first term of Eq. (1) can be written as an integral over the cell of an energy density $\mathcal{E}_{\text {Sky }}(\boldsymbol{r})$, thus

$$
e_{\text {Sky }}=\frac{1}{A} \int_{\text {cell }} \mathcal{E}_{\text {Sky }}(\boldsymbol{r}) d^{3} \boldsymbol{r}
$$


in the case of cylindrical shapes the integration is taken over unit length and in the case of plates over unit area. For our generalized Skyrme functionals the energy density $\mathcal{E}_{\text {Sky }}(\boldsymbol{r})$ is given by Eq. (A3) of Ref. [12] in terms of the number densities $n_{q}(\boldsymbol{r})$, the kinetic-energy densities $\tau_{q}(\boldsymbol{r})$ and the spin-current densities $\boldsymbol{J}_{q}(\boldsymbol{r})$, where $q=p$ or $n$ denotes protons or neutrons, respectively.

In the first stage of the calculation of the energy per nucleon (1) the term $e_{\text {Sky }}$ is evaluated by the ETF method, which consists in approximating $\tau_{q}(\boldsymbol{r})$ and $\boldsymbol{J}_{q}(\boldsymbol{r})$ as functions of the number densities $n_{q}(\boldsymbol{r})$ and their derivatives. To speed up the computations we avoided having to solve the Euler-Lagrange equations by parametrizing the neutron and proton density distributions $n_{q}(\boldsymbol{r})$ in both Refs. [2,10] according to

$$
\tilde{n_{q}}(\xi)=n_{B q}+n_{\Lambda q} f_{q}(\xi)
$$

in which the first term represents a constant background and the second a cluster term with

$$
f_{q}(\xi)=\frac{1}{1+\exp \left[\left(\frac{C_{q}-R}{\xi-R}\right)^{2}-1\right] \exp \left(\frac{\xi-C_{q}}{a_{q}}\right)} .
$$

Here $\xi$ denotes the radial coordinate $r$ in the case of spherical cells, the radial coordinate $\eta$ in the case of cylindrical cells, and the Cartesian coordinate $z$ for plates, assumed to lie in the $x-y$ plane. The parameter $R$ likewise represents the radius of the spherical cell, the radius of the cylindrical cell or the semithickness of the platelike cell. The ETF approximation to the energy per nucleon (1) thus reads

$$
\widetilde{e}=\widetilde{e_{\mathrm{Sky}}}+e_{C}+e_{e}-Y_{p} Q_{n, \beta},
$$

where $\widetilde{e_{\text {Sky }}}$ is the ETF approximation to $e_{\text {Sky }}$. Then for given mean density $\bar{n}$ and $Z$ the estimate $\widetilde{e}$ is optimized with respect to all the parameters of Eq. (4) and the neutron number $N$.

In adopting the ETF approximation all shell effects are inevitably lost, but in the spherical-cell calculations of Ref. [2] they were restored perturbatively for protons by the SI method; pairing was taken into account using the BCS method. We then had for our improved approximation to the energy per nucleon (1)

$$
e_{\mathrm{ETFSI}}=\widetilde{e}+\frac{1}{A}\left(E_{p}^{\mathrm{SI}, \text { pair }}+E_{p}^{\mathrm{pair}}\right)
$$

in which $E_{p}^{\mathrm{SI} \text {,pair }}$ is the SI correction for protons, as modified by pairing, and $E_{p}^{\text {pair }}$ is the proton BCS energy [7].

The argument proffered in Ref. [10] for neglecting proton shell corrections (and, by implication, pairing corrections) in pasta ran as follows. In the case of both spaghetti and lasagna the motion of protons in at least one dimension is unbounded (along the symmetry axis in the former case and in the plane of the plate in the latter case), which means that the proton s.p. states form a continuum (the situation is actually a little more complicated; see Sec. II B). This certainly implies that there will be no characteristic shell-model fluctuations in the energy per nucleon as the number of protons in the WS cell varies, and we took this to mean that the SI corrections should vanish.

Let us, however, reexamine the derivation of the SI theorem given in Appendix C of Ref. [5] as an approximation to the
HF method. From the ETF proton-density distribution $\tilde{n_{p}}(\boldsymbol{r})$ emerging from the ETF stage of the calculation and the corresponding ETF neutron-density distribution we can generate the nuclear (Skyrme) s.p. central and spin-orbit fields, $\widetilde{U}_{p}(\boldsymbol{r})$ and $\widetilde{\boldsymbol{W}}_{\boldsymbol{p}}(\boldsymbol{r})$, respectively, the Coulomb field $\widetilde{U_{C}}(\boldsymbol{r})$ and the effective proton mass $\widetilde{M}_{p}^{*}(\boldsymbol{r})$. The corresponding proton s.p. Schrödinger equation is then

$$
\begin{aligned}
& \left\{-\nabla \frac{\hbar^{2}}{2 \widetilde{M}_{p}^{*}(\boldsymbol{r})} \cdot \nabla+\widetilde{U}_{p}(\boldsymbol{r})+\widetilde{U}_{C}(\boldsymbol{r})-i{\widetilde{\boldsymbol{W}_{p}}}_{\boldsymbol{p}}(\boldsymbol{r}) \cdot \nabla \times \boldsymbol{\sigma}\right\} \psi_{\nu, p} \\
& =\widetilde{\epsilon}_{v, p} \psi_{v, p} .
\end{aligned}
$$

Henceforth we drop the superfluous proton subscript $p$ on the s.p. eigenfunctions $\psi_{v, p}$ and the s.p. eigenvalues $\widetilde{\epsilon}_{v, p}$; also we will write the proton density distribution corresponding to the s.p. eigenfunctions $\psi_{v}$ as $n_{p}^{\prime}(\boldsymbol{r})$. We next write the exact HF proton density, constrained to have the appropriate pasta symmetry, as

$$
n_{p}(\boldsymbol{r})=\tilde{n_{p}}(\boldsymbol{r})+\delta n_{p}(\boldsymbol{r}) .
$$

The essence of the SI method is to write now

$$
\delta n_{p}(\boldsymbol{r})=\left[n_{p}^{\prime}(\boldsymbol{r})-\tilde{n_{p}}(\boldsymbol{r})\right]+\left[n_{p}(\boldsymbol{r})-n_{p}^{\prime}(\boldsymbol{r})\right],
$$

and then drop the second term, an approximation that amounts to assuming that the HF method is sufficiently converged after one iteration, using the ETF solution as the initial guess. This essentially first-order perturbative treatment, suitably extended to include BCS pairing [7], then leads to Eq. (6) with

$$
\begin{aligned}
E_{p}^{\text {SI,pair }}= & \sum_{v} V_{v}^{2} \widetilde{\epsilon}_{v}-\int d^{3} \boldsymbol{r}\left\{\frac{\hbar^{2}}{2 \widetilde{M}_{p}^{*}(\boldsymbol{r})} \widetilde{\tau_{p}}(\boldsymbol{r})\right. \\
& \left.+{\widetilde{n_{p}}}_{(\boldsymbol{r})}\left[\widetilde{U}_{p}(\boldsymbol{r})+\widetilde{U_{C}}(\boldsymbol{r})\right]+\widetilde{\boldsymbol{J}_{p}}(\boldsymbol{r}) \cdot \widetilde{\boldsymbol{W}_{\boldsymbol{p}}}(\boldsymbol{r})\right\},
\end{aligned}
$$

where $V_{v}$ denotes the BCS occupation factor (see Ref. [7] for details).

Any shell-model fluctuations associated with varying proton number $Z$ will be manifested in the first term of Eq. (10), the Strutinsky sum, and if the s.p. states form a continuum such fluctuations must vanish. But even in that case there is no $a$ priori reason why the smoothly varying second term of Eq. (10), the Strutinsky integral, should be exactly equal to the smoothly varying first term. Any difference between the two must originate in the term in Eq. (9) that is retained, $n_{p}^{\prime}(\boldsymbol{r})-\tilde{n_{p}}(\boldsymbol{r})$, which can never vanish identically at all points in the cell, since $\tilde{n_{p}}(\boldsymbol{r})$ is a smoothly varying function of $r$ while $n_{p}^{\prime}(\boldsymbol{r})$ fluctuates. Indeed, it could be large if the ETF distribution $\tilde{n_{p}}(\boldsymbol{r})$ differs significantly from the final HFB distribution $n_{p}(\boldsymbol{r})$, assuming that the iterated density $n_{p}^{\prime}(\boldsymbol{r})$ is appreciably closer to $n_{p}(\boldsymbol{r})$ [if it is not then the neglected term in Eq. (9) will not be negligible, and the method fails anyway]. More generally, the SI correction is more than just a shell correction: It will consist of two terms distinguished by their behavior as a function of $Z$ at a given mean density $\bar{n}$ : a term fluctuating about zero and a smoothly varying term. Only the first of these terms is a true shell correction; the second is a correction for deviations between the ETF distribution and the smoothed version of the exact distribution that would have 


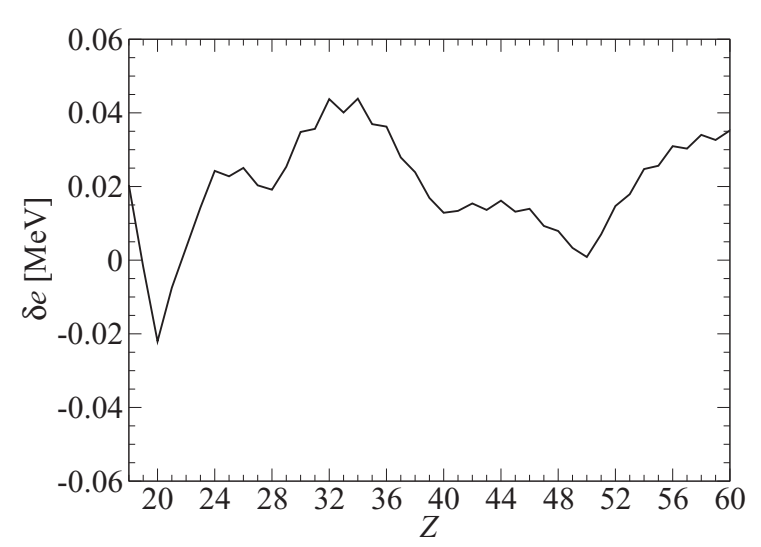

FIG. 1. The microscopic (SI plus pairing) correction (in MeV) as a function of $Z$ for functional BSk24 with spherical WS cells at $\bar{n}=2.7 \times 10^{-4} \mathrm{fm}^{-3}$. The neutron number $N$ is optimized for each $Z$.

been found in a complete HFB calculation (it was just the first term that was estimated in the pioneer work of Ref. [13]).

There are two sources of deviations between the ETF distribution and the smoothed HF distribution. The first arises from the general tendency of the ETF method to overbind with respect to smoothed HF calculations (see, for example, Section 4 of Ref. [14]). The second has the opposite sign and comes from our decision to parametrize the ETF distributions rather than solve the Euler-Lagrange equations; it will inevitably lead to a tendency to underbind.

A striking example of a significant smooth contribution to the SI correction for protons is already available in the spherical-cell calculations of Ref. [2], as shown in Fig. 1 for a density of $2.7 \times 10^{-4} \mathrm{fm}^{-3}$, close to the interface with the outer crust. Although the SI correction shows a clear-cut shell structure, with magic numbers at the familiar values of 20,28 , 40 ("semimagic") and 50, its average value is far from zero; in fact, it is positive for virtually all values of $Z$. This means that the inherent tendency of the ETF method to overbind is more significant than any error in the choice of parametrization, at least in this case.

Having made it amply clear that even in the anticipated absence of shell fluctuations, we cannot exclude significant SI corrections, we are obliged to repeat our pasta calculations of Ref. [10]. However, the argument that we made above for neglecting the neutron SI correction, citing Ref. [5], is valid only for the fluctuating part, the true shell correction; it does not apply to the smoothly varying part. To address this question we note that the neutrons, being some 30 times more numerous than the protons in the inner crust, are distributed far more homogeneously than the protons. This can be seen from Table XI of Ref. [10], where we show the nucleonic inhomogeneity factor

$$
\Lambda=\frac{1}{V_{\text {cell }}} \int\left[\frac{n(\boldsymbol{r})}{\bar{n}}-1\right]^{2} d^{3} \boldsymbol{r}
$$

and the purely protonic inhomogeneity factor

$$
\Lambda_{p}=\frac{1}{V_{\text {cell }}} \int\left[\frac{n_{p}(\boldsymbol{r})}{\bar{n}_{p}}-1\right]^{2} d^{3} \boldsymbol{r},
$$

in which $V_{\text {cell }}$ is the cell volume: $\Lambda_{p}$ is around 40 times greater than $\Lambda$ in the inner crust, and the ratio between $\Lambda_{p}$ and $\Lambda_{n}$ will be even greater. We will now argue that the much greater homogeneity of the neutron distribution means that both contributions to the smoothly varying part of the SI correction will be much smaller for the neutrons than for the protons.

First, since the intrinsic difference between smoothed HF and ETF vanishes in the limit of homogeneous matter the characteristic overbinding of ETF will be much less significant for neutrons than for protons. Concerning the separate question of an inadequate parametrization, we note that the one that we have adopted, as given by Eqs. (3) and (4), is far from being the most general imaginable, but any changes can only be made in the cluster term $f_{q}(\xi)$. However, the significance of this term relative to the constant background will be smaller the more homogeneous the distribution.

This justification of our neglect of the neutron SI correction applies equally well to our neglect of the proton SI correction once proton drip sets in, close to the interface between the inner crust and the core [2].

The plan of the present paper is as follows: The SI formalism for pasta is described in Sec. II B and our treatment of pairing in Sec. II A, while our results for functional BSk24 are presented in Sec. III. We summarize our conclusions in Sec. IV.

\section{FORMALISM}

\section{A. Treatment of pairing}

Since we are dealing with weakly inhomogeneous configurations at densities $\bar{n} \gtrsim 0.05 \mathrm{fm}^{-3}$ close to the crust-core transition $\bar{n}_{\mathrm{cc}} \sim 0.08 \mathrm{fm}^{-3}$, we adopt the local density approximation for the condensation energy, as in Ref. [9], instead of our usual implementation of the BCS method [7], as in our earlier EoS calculations [2]. Thus Eq. (6) is replaced by

$$
e_{\mathrm{ETFSI}}=\widetilde{e}+\frac{1}{A}\left(E_{p}^{\mathrm{SI}}+E_{p}^{\mathrm{pair}}\right),
$$

in which $E_{p}^{\mathrm{SI}}$ is the SI correction without any modification for pairing, i.e., Eq. (10) is replaced by

$$
\begin{aligned}
E_{p}^{\mathrm{SI}}= & \sum_{\nu(\mathrm{occ})} \widetilde{\epsilon}_{\nu}-\int d^{3} \boldsymbol{r}\left\{\frac{\hbar^{2}}{2{\widetilde{M_{p}^{*}}}^{*}(\boldsymbol{r})} \widetilde{\tau_{p}}(\boldsymbol{r})+\widetilde{n_{p}}(\boldsymbol{r})\right. \\
& \left.\times\left[{\widetilde{U_{p}}}_{p}(\boldsymbol{r})+{\widetilde{U_{C}}}_{C}(\boldsymbol{r})\right]+\widetilde{\boldsymbol{J}}_{p}(\boldsymbol{r}) \cdot{\widetilde{\boldsymbol{W}_{p}}}_{p}(\boldsymbol{r})\right\},
\end{aligned}
$$

in which the summation only goes over the occupied s.p. states.

Also in Eq. (12), $E_{p}^{\text {pair }}$ is now determined by adding to the energy density, after optimization of the ETF part of the calculation,

$$
\mathcal{E}_{\text {cond }, p}(\boldsymbol{r})=-\frac{3}{8} n_{p}(\boldsymbol{r}) \frac{\Delta_{p}(\boldsymbol{r})^{2}}{\epsilon_{F}(\boldsymbol{r})},
$$

whence

$$
E_{p}^{\text {pair }}=\int d^{3} \boldsymbol{r} \mathcal{E}_{\text {cond }, p}(\boldsymbol{r})
$$


Here $\Delta_{p}(\boldsymbol{r})$ is the proton pairing gap of homogeneous nuclear matter of the appropriate density and charge asymmetry, as determined by many-body calculations with realistic two- and three-body interactions. The latter gaps, on which the adopted BSk24 functional is based, were parametrized as described in Ref. [15]. Also, $\epsilon_{F}(\boldsymbol{r})$ is the local Fermi energy,

$$
\epsilon_{F}(\boldsymbol{r})=\frac{\hbar^{2}}{2 M_{p}}\left[3 \pi^{2} n_{p}(\boldsymbol{r})\right]^{2 / 3} .
$$

\section{B. Strutinsky-integral theorem for pasta}

The s.p. equation (7) has to be solved for both cylindrical and platelike configurations. To avoid great complication we make the approximation of replacing the position-dependent proton effective mass $\widetilde{M}_{p}^{*}(\boldsymbol{r})$ by the real mass $M_{p}$. To avoid internal inconsistencies, the effective mass is absorbed into the potential $U_{p}(\boldsymbol{r})$ by replacing the proton kinetic density $\tau_{p}(\boldsymbol{r})$ in the potential part of the Skyrme energy density $\mathcal{E}_{\text {Sky }}(\boldsymbol{r})$ by its TF approximation,

$$
\tau_{p}(\boldsymbol{r})=\frac{3}{5}\left(3 \pi^{2}\right)^{2 / 3} n_{p}(\boldsymbol{r})^{5 / 3} .
$$

In this way, the s.p. proton central field, defined by

$$
U_{p}(\boldsymbol{r})=\frac{\partial \mathcal{E}_{\mathrm{Sky}}(\boldsymbol{r})}{\partial n_{p}(\boldsymbol{r})}-\nabla \cdot \frac{\partial \mathcal{E}_{\mathrm{Sky}}(\boldsymbol{r})}{\partial\left[\nabla n_{p}(\boldsymbol{r})\right]},
$$

and given by Eq. (A11) of Ref. [12], now includes an additional contribution due to the density dependence of $\tau_{p}$, namely

$$
U_{p}^{*}(\boldsymbol{r})=\left[\frac{\hbar^{2}}{2 \widetilde{M_{p}^{*}(\boldsymbol{r})}}-\frac{\hbar^{2}}{2 M_{p}}\right] k_{F p}(\boldsymbol{r})^{2},
$$

where we have introduced the local proton Fermi wave number $k_{F p}=\left(3 \pi^{2} n_{p}\right)^{1 / 3}$ and $\widetilde{M}_{p}^{*}(\boldsymbol{r})$ is the proton effective mass that appears in Eq. (7) and is given by Eq. (A10) of Ref. [12]. Testing this approximation on spherical WS cells shows that it can affect the 4th significant figure, a shift that is large enough to change the optimum shape. Thus in comparing pasta shapes with spherical shapes the latter must be calculated with this approximation.

Again in order to simplify the calculations, we drop the spin-orbit term in the s.p. field (but not in the ETF part of the calculation). We have tested the validity of this approximation on spherical WS cells at densities where pasta configurations are typically found, i.e., close to the interface with the core. Differences arise only in the sixth significant figure, being smaller than $30 \mathrm{eV}$. The negligibility of the spin-orbit contribution is simply a consequence of the near-homogeneity at these densities.

\section{Spaghetti}

In the case of cylinders we consider eigensolutions to Eq. (7) of the separated form

$$
\psi_{\nu}(\boldsymbol{r})=F_{\nu}(\eta) \exp (i m \phi) \exp \left(i k_{z} z\right),
$$

where $m$ is an integer and we are using cylindrical coordinates with the symmetry axis taken along the $z$ axis. Then, dropping all the tildes, Eq. (7) reduces to

$$
\begin{aligned}
& \frac{d^{2} F_{v}}{d \eta^{2}}+\frac{1}{\eta} \frac{d F_{v}}{d \eta} \\
& \quad+\left\{\frac{2 M_{p}}{\hbar^{2}}\left[\epsilon_{v}-U_{p}(\eta)-U_{C}(\eta)\right]-k_{z}^{2}-\frac{m^{2}}{\eta^{2}}\right\} F_{v}=0 .
\end{aligned}
$$

We now write

$$
\epsilon_{v}=e_{\mu}+\frac{\hbar^{2} k_{z}^{2}}{2 M_{p}}
$$

where $\mu \equiv(j, m)$, in which $j$ represents all quantum numbers other than $m$ and $k_{z}$ characterizing the eigenfunction, i.e., $v \equiv$ $\left(j, m, k_{z}\right)$. Then Eq. (21) becomes

$$
\begin{aligned}
& \frac{d^{2} F_{\mu}(\eta)}{d \eta^{2}}+\frac{1}{\eta} \frac{d F_{\mu}(\eta)}{d \eta} \\
& +\left\{\frac{2 M_{p}}{\hbar^{2}}\left[e_{\mu}-U_{p}(\eta)-U_{C}(\eta)\right]-\frac{m^{2}}{\eta^{2}}\right\} F_{\mu}(\eta)=0 .
\end{aligned}
$$

The nuclear (Skyrme) field $U_{p}(\eta)$ is given directly by Eq. (A11) of Ref. [12], but note that the terms in $J^{2}$ and $J_{q}^{2}$ are dropped in the BSk24 functional in order to avoid spurious spin and spin-isospin instabilities [16]. For the Coulomb field we use the expression (see Appendix)

$$
U_{C}(\eta)=4 \pi e^{2} \int_{\eta}^{R} \ln \left(\frac{\eta}{\eta^{\prime}}\right) n_{\mathrm{ch}}\left(\eta^{\prime}\right) \eta^{\prime} d \eta^{\prime},
$$

valid for an axially symmetric charge distribution $n_{\mathrm{ch}}\left(\eta^{\prime}\right)$ (in units of the elementary electric charge $e$ ) in a globally neutral infinitely long cylinder of radius $R$.

Equation (23) is an eigenequation describing the transverse motion, i.e., the motion in the $x-y$ plane. The corresponding eigenstates constitute a discrete spectrum, characteristic of spaghetti; the eigenvalues $e_{\mu}$ are determined by expanding the solutions $F_{\mu}(\eta)$ on a basis of cylindrical Bessel functions $J_{m}\left(k_{i} \eta\right)$ in which the values of $k_{i}$ are determined for each quantum number $m$ by imposing suitable boundary conditions. Assuming pastas are regularly arranged, the eigenfunctions must be of the Bloch form. However, since the microscopic corrections are only calculated for bound protons, we can alternatively impose homogeneous boundary conditions, the choice for which is somewhat arbitrary. If we require that all the basis functions vanish at the cell surface, $\eta=R$, then obviously the corresponding density will be too low, while if we require that it is their derivatives that vanish at the cell surface then the density will be too high. We therefore adopt the compromise solution of Negele and Vautherin (NV) [17], i.e., the basis functions vanish at the cell surface for $m$ even, while their derivatives vanish at the cell surface for $m$ odd. But from this standpoint the "anti-NV" choice of boundary conditions, in which the basis functions vanish at the cell surface for $m$ odd, while their derivatives vanish at the cell surface for $m$ even, would be equally satisfactory [18]. We find that making this choice for cylinders will affect the energy per nucleon only in the sixth figure at densities of 
around $\bar{n}=5.6 \times 10^{-2} \mathrm{fm}^{-3}$, which is quite insignificant, but at higher densities of around $\bar{n}=7.1 \times 10^{-2} \mathrm{fm}^{-3}$ the fourth figure can be affected. However, we find that our spherical code has a similar sensitivity to the boundary conditions (a fact that we have not previously reported) and thus conclude that the sphere-cylinder transition is insensitive to the choice of boundary conditions.

Taking the longitudinal motion, i.e., the motion along the $z$ axis, into account, the states (20) are seen to constitute a set of continuous spectra, each one based on one or other of the discrete transverse states $\mu$ having eigenenergy $e_{\mu}$. This clean separation into states of transverse and states of longitudinal motion is possible only because of the approximation $M_{p}^{*}=M_{p}$ that we have made, and because we have dropped the spin-orbit term. (There will be a discontinuous change in the degeneracy as continuum longitudinal states based on a new discrete transverse state begin to be filled, and this might be expected to lead to a weak shell structure.)

The Strutinsky sum, i.e., the first term of Eq. (13), thus becomes an integral, or rather a sum of integrals. To evaluate these integrals we require the state density of the continua. Suppose that the cylinders have finite length $L$ rather than being infinitely long. Then requiring vanishing wave functions at the two extremities means that only values of $k_{z}$ given by

$$
k_{z}=\frac{n \pi}{L}
$$

where $n$ is integral, are allowed. It follows that the number of states all based on the same transverse state $\mu$ with longitudinal quantum number lying between $k_{z}$ and $k_{z}+d k_{z}$ is $(L / \pi) d k_{z}$. Thus the number of states per unit length over interval $d k_{z}$ is $d k_{z} / \pi$, regardless of the value of $L$, whence the number of states per unit length up to a maximum value of $k_{z}=k_{F, \mu}$ will be $k_{F, \mu} / \pi$, for a given transverse state $\mu$. Now the maximum value of $k_{z}$ for a given transverse state $\mu$ at zero temperature, which we assume throughout this paper, is given, according to Eq. (22), by

$$
\frac{\hbar^{2}}{2 M_{p}} k_{F, \mu}^{2}=\epsilon_{F}-e_{\mu},
$$

where $\epsilon_{F}$ is the proton Fermi energy of the cell. The latter, not to be confused with the quantity defined in Eq. (16), is given in terms of the number of protons per unit length by

$$
Z_{l}=\frac{2}{\pi}\left(\frac{2 M_{p}}{\hbar^{2}}\right)^{1 / 2} \sum_{\mu}\left(\epsilon_{F}-e_{\mu}\right)^{1 / 2},
$$

where we have admitted two protons to each spatial state to account for the spin degeneracy. To solve this equation we used the brute-force bisection method, the Newton-Raphson method proving to be unstable; the sum has to be performed over all transverse states $\mu$, i.e., over all $(j, m)$, for which the summand is real.

The energy per unit length is now given by

$$
\begin{aligned}
E_{l} & =\frac{2}{\pi} \sum_{\nu} \int_{0}^{k_{F, \mu}}\left(e_{\mu}+\frac{\hbar^{2}}{2 M_{p}} k_{z}^{2}\right) d k_{z} \\
& =\frac{2}{3 \pi}\left(\frac{2 M_{p}}{\hbar^{2}}\right)^{1 / 2} \sum_{\mu}\left(\epsilon_{F}-e_{\mu}\right)^{1 / 2}\left(\epsilon_{F}+2 e_{\mu}\right) .
\end{aligned}
$$

\section{Lasagna}

For plates we consider eigensolutions to Eq. (7) of the separated form

$$
\psi_{\nu}(\boldsymbol{r})=G_{v}(z) \exp \left(i k_{x} x+i k_{y} y\right),
$$

where we take the plates to be lying in the $x-y$ plane, with the cell surfaces lying at $z= \pm R$. Equation (7) then reduces to

$$
\begin{aligned}
& \frac{d^{2} G_{v}(z)}{d z^{2}} \\
& \quad+\left\{\frac{2 M_{p}}{\hbar^{2}}\left[\epsilon_{v}-U_{p}(z)-U_{C}(z)\right]-\left(k_{x}^{2}+k_{y}^{2}\right)\right\} G_{\nu}(z)=0 .
\end{aligned}
$$

We now write

$$
\epsilon_{v}=e_{\mu}+\frac{\hbar^{2}}{2 M_{p}}\left(k_{x}^{2}+k_{y}^{2}\right),
$$

where $\mu$ is the single quantum number relating to longitudinal motion, i.e., motion along the $z$ axis. Then Eq. (30) becomes

$$
\frac{d^{2} G_{\mu}(z)}{d z^{2}}+\left\{\frac{2 M_{p}}{\hbar^{2}}\left[e_{\mu}-U_{p}(z)-U_{C}(z)\right]\right\} G_{\mu}(z)=0 .
$$

Again, the nuclear (Skyrme) field $U_{p}(\eta)$ is given directly by Eq. (A11) of Ref. [12], while for the Coulomb field we use the expression (see Appendix)

$$
U_{C}(z)=4 \pi e^{2} \int_{z}^{R}\left(z-z^{\prime}\right) n_{\mathrm{ch}}\left(z^{\prime}\right) d z^{\prime},
$$

assuming global neutrality of the WS cell.

The eigenstates $G_{\mu}(z)$, describing longitudinal motion, constitute a discrete spectrum, characteristic of lasagna. The eigenvalues $e_{\mu}$ are determined by expanding the solutions $G_{\mu}(z)$ on a basis of trigonometric functions, subjected to homogeneous boundary conditions on the cell surfaces at $z= \pm R$. There are two obvious choices for our basis set,

$$
\phi_{n}(z)=\sin \frac{n \pi}{2 R}(z+R)
$$

and

$$
\phi_{n}(z)=\cos \frac{n \pi}{2 R}(z+R) .
$$

The first of these vanishes at the cell surfaces, while for the second it is derivatives that vanish there. We quite arbitrarily adopted the second option, but tests showed that only the sixth significant figure of the total energy per nucleon is sensitive to this choice, which is within the limits of our overall numerical accuracy. Because only one set of basis functions enters into these one-dimensional calculations it is not possible to make a choice of boundary conditions comparable to those of NV [17]. Taking the motion in the $x-y$ plane into account, we see that with the states (29) we once again are dealing with a set of continuous spectra, each one based this time on one or other of the discrete longitudinal states having eigenenergy $e_{\mu}$. (As in the case of spaghetti, a weak shell structure might be expected to arise as continuum transverse states associated with a new discrete longitudinal state begin to be filled.) 
The Strutinsky sum is again a sum of integrals for the evaluation of which we require the state density of the continua. Let us imagine that there are walls at $x= \pm L / 2, y= \pm L / 2$ on which the wave functions must vanish. Then $k_{\perp}^{2} \equiv k_{x}^{2}+k_{y}^{2}$ can take only the values given by

$$
k_{\perp}^{2}=\frac{\pi^{2}}{L^{2}}\left(n^{2}+m^{2}\right),
$$

where $m$ and $n$ are integers. Rewriting this last equation as

$$
\rho^{2} \equiv n^{2}+m^{2}=\frac{L^{2}}{\pi^{2}} k_{\perp}^{2},
$$

it follows that the number of states all based on the same longitudinal state $\mu$ with transverse quantum number lying between $k_{\perp}$ and $k_{\perp}+d k_{\perp}$ is

$$
\frac{1}{2} \pi \rho d \rho=\frac{L^{2}}{2 \pi} k_{\perp} d k_{\perp} .
$$

Thus the number of states per unit area over the interval $k_{\perp}$ and $k_{\perp}+d k_{\perp}$ is $k_{\perp} d k_{\perp} /(2 \pi)$, regardless of the value of $L$, and the number per unit area for a given longitudinal state $\mu$ up to a maximum value of $k_{\perp}=k_{F, \mu}$ will be

$$
\frac{1}{2 \pi} \int_{0}^{k_{F, \mu}} k_{\perp} d k_{\perp}=\frac{1}{4 \pi} k_{F, \mu}^{2} .
$$

Now the maximum value of $k_{\perp}$ for a given longitudinal state $\mu$ is given by

$$
k_{F, \mu}^{2}=\frac{2 M_{p}}{\hbar^{2}}\left(\epsilon_{F}-e_{\mu}\right),
$$

where we have used Eq. (31). Then with two protons per spatial state the total number of protons per unit area is

$$
Z_{s}=\frac{1}{2 \pi} \frac{2 M_{p}}{\hbar^{2}} \sum_{\mu}\left(\epsilon_{F}-e_{\mu}\right),
$$

which can be solved for $\epsilon_{F}$ in terms of $Z_{s}$ as in the case of spaghetti.

The energy per unit area is now given by

$$
\begin{aligned}
E_{s} & =\frac{1}{\pi} \sum_{\mu} \int_{0}^{k_{F, \mu}}\left(e_{\mu}+\frac{\hbar^{2}}{2 M_{p}} k_{\perp}^{2}\right) k_{\perp} d k_{\perp} \\
& =\frac{1}{2 \pi} \frac{M_{p}}{\hbar^{2}} \sum_{\mu}\left(\epsilon_{F}^{2}-e_{\mu}^{2}\right),
\end{aligned}
$$

where in the second step we have used Eq. (39).

\section{Proton drip}

In Ref. [2] our criterion for determining the proton drip point involved the kinetic-energy density at the center of the cell, and for this latter quantity we used the simple TF expression. It turned out that for cylinders this was not accurate enough and that serious errors could arise. We therefore adopted as the criterion for proton drip the condition that the proton Fermi energy $\epsilon_{F}$ should exceed the total proton s.p. field at the cell surface,

$$
\epsilon_{F} \geqslant U_{p}(R)+U_{C}(R) .
$$

\section{RESULTS FOR FUNCTIONAL BSK24}

Our principal results are shown in Tables I and II, with full details being found in electronic form in the Supplemental Material [19]. In Fig. 2 we show for the typical density of $\bar{n}=$ $0.0738136 \mathrm{fm}^{-3}$ the ETF and ETFSI energies for the three possible cell forms. No shell structure in the SI correction is apparent, not even one associated with weakly discontinuous changes in the degeneracy of the kind discussed in Sec. II B: The SI corrections for pasta are entirely smoothly varying.

\section{A. Equilibrium shape}

Table I shows the energy per nucleon $e$, optimized with respect to $N, Z$ and the geometrical parameters of Eqs. (3) and (4), at a number of different densities $\bar{n}$ selected over the full range for which pasta solutions are found. The optimization has been performed by repeating the calculations over a wide range of fixed values of $Z$, at each one of which there has been automatic minimization with respect to $N$ and the geometrical parameters. Each of these calculations is performed with spherical, cylindrical and plate cell shapes imposed in turn.

There are two columns in Table I for each of the three cell shapes, the first, labeled "(1)," being pure ETF, and the second, labeled "(2)," including the microscopic corrections (SI and pairing). The pure ETF column for spherical cells, "sph(1)," has simply been copied from Table I of Ref. [10], while for the column "sph(2)" the microscopic corrections have been recalculated adopting the same approximations as for the pasta calculations. That is, the position-dependent proton effective mass $\widetilde{M_{p}^{*}}(\boldsymbol{r})$ is replaced by the real mass $M_{p}$, with suitable adjustment of the proton mean field $U_{p}(\boldsymbol{r})$, and the pairing correction is calculated in the local-density approximation, rather than in BCS. In this way, meaningful comparisons can be made. The two pasta ETF columns, "cyl(1)" and "plate(1)," are essentially the same as in Table I of Ref. [10], with some minor changes. The last two columns indicate the equilibrium shape for the pure ETF calculations and the ETFSI + pairing calculations, respectively; they are determined by comparing the corresponding values of $e$ for the three different cell shapes.

The asterisks in the columns " $\operatorname{sph}(2)$ " and "cyl(2)" indicate the density $\bar{n}_{\text {pd }}$ beyond which proton drip, as determined by Eq. (42), occurs at the equilibrium value of $Z$. Actually, since there will be proton drip at all densities if $Z$ is large enough, we have at every density to calculate for increasing values of $Z$ up to and beyond the value at which proton drip occurs. Before the drip value of $Z$ is reached we invariably find that the ETFSI value of $e$ passes through a minimum, but this is not necessarily the equilibrium point, since once drip has set in our model requires us to drop the microscopic corrections (see Sec. I). In fact, a lower minimum will be found at some higher value of $Z$ if $\bar{n} \geqslant \bar{n}_{\text {pd }}$ but not otherwise. For cylindrical shapes over the density range $\bar{n}_{\text {pd }} \leqslant \bar{n} \leqslant 0.0703677 \mathrm{fm}^{-3}$ the energy per nucleon $e$ takes a higher value in the column "cyl(2)" than in "cyl(1)." This is because over this density range the minimum value of $e$ without the inclusion of microscopic corrections occurs at a subdrip value of $Z$. 
TABLE I. Energy per nucleon (in MeV) as function of density $\bar{n}\left(\right.$ in $\mathrm{fm}^{-3}$ ) for different cell shapes; $\operatorname{sph}(1)$ gives equilibrium ETF value for spherical shape, $\operatorname{sph}(2)$ gives equilibrium ETFSI + pairing value for spherical shape. Likewise for cyl(1), cyl(2), plate(1), and plate(2). Optimal shapes denoted by s (spherical), c (cylindrical), and p (platelike); shape(1) gives ETF, shape(2) gives ETFSI + pairing. Asterisk denotes onset of proton drip (SI + pairing corrections set equal to zero).

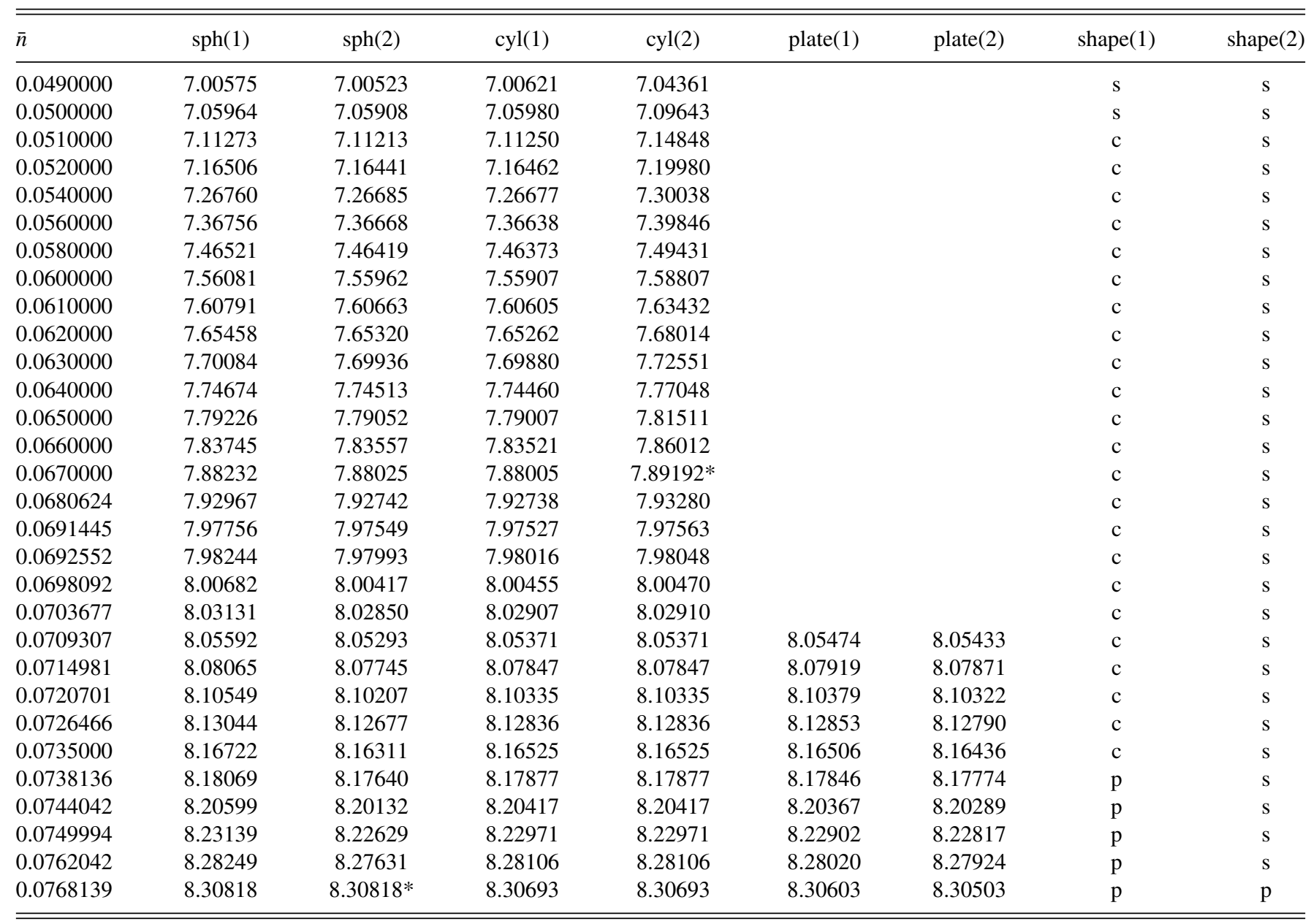

Table I shows us that with pure ETF calculations the cell shape evolves as the density increases in the usual sequence sphere $\rightarrow$ cylinder $\rightarrow$ plate before the transition to the homogeneous core. In fact, as we discussed in Ref. [10], the densities associated with transitions to different shapes in a pure ETF treatment follow closely those predicted by the liquid-drop approach [20]. However, including the microscopic corrections has the dramatic effect of almost completely eliminating the pasta phases: There is no more spaghetti, and the WS cells remain spherical almost up to the interface with the homogeneous core, where a thin layer of lasagna puts in an appearance. Moreover, the transition from spheres to plates at $\bar{n}=0.0768139 \mathrm{fm}^{-3}$ is entirely a consequence of proton drip having set in at this density for spherical cells. If we had not simply dropped the microscopic corrections in this situation and had instead adopted a more sophisticated treatment of continuum states, then it is conceivable that the WS cells would have retained their spherical form right up to the interface with the homogeneous core.

It can be seen from Table I that the absence of a spaghetti phase results from the microscopic corrections for cylinders being positive, while those for spheres and plates are neg- ative. (Note that since the equilibrium values of $N, Z$ and the geometrical parameters depend on whether or not the microscopic corrections are included, the difference between the two columns in Table I for a given shape and density does not represent exactly the value of the microscopic correction for any given configuration, but it nevertheless gives a reliable notion of their trends. For a more precise picture the supplemental material should be consulted.) Even though the positive corrections for cylinders are quite large, they are not abnormally so, as a comparison of Figs. 1 and 2 will make clear. In any case, even if the (positive) microscopic corrections for cylinders were reduced to be no larger in magnitude than the (negative) microscopic corrections for spheres there would still be no spaghetti (note that for $\bar{n}>0.067 \mathrm{fm}^{-3}$ there are no corrections to be made in the case of cylinders, because of proton drip). Moreover, the fact that at higher densities replacing the NV boundary conditions by the "anti$\mathrm{NV}$ " conditions raises the energy per nucleon of cylinders in the fourth significant figure while the energies of spheres and plates are quite insensitive to the choice of boundary conditions makes the appearance of spaghetti still more unlikely. It is worth noting that even for cylinders the corrections only 
TABLE II. Equilibrium parameters: proton fraction $Y_{p}$, pressure $P\left(\mathrm{MeV} \mathrm{fm}^{-3}\right)$, proton number $Z$ (units depend on shape: see text) and shape (s, spherical; c, cylindrical; and p, platelike), as functions of density $\bar{n}$ (in $\mathrm{fm}^{-3}$ ). Columns labeled "1" for pure ETF, columns labeled "2" for ETFSI + pairing.

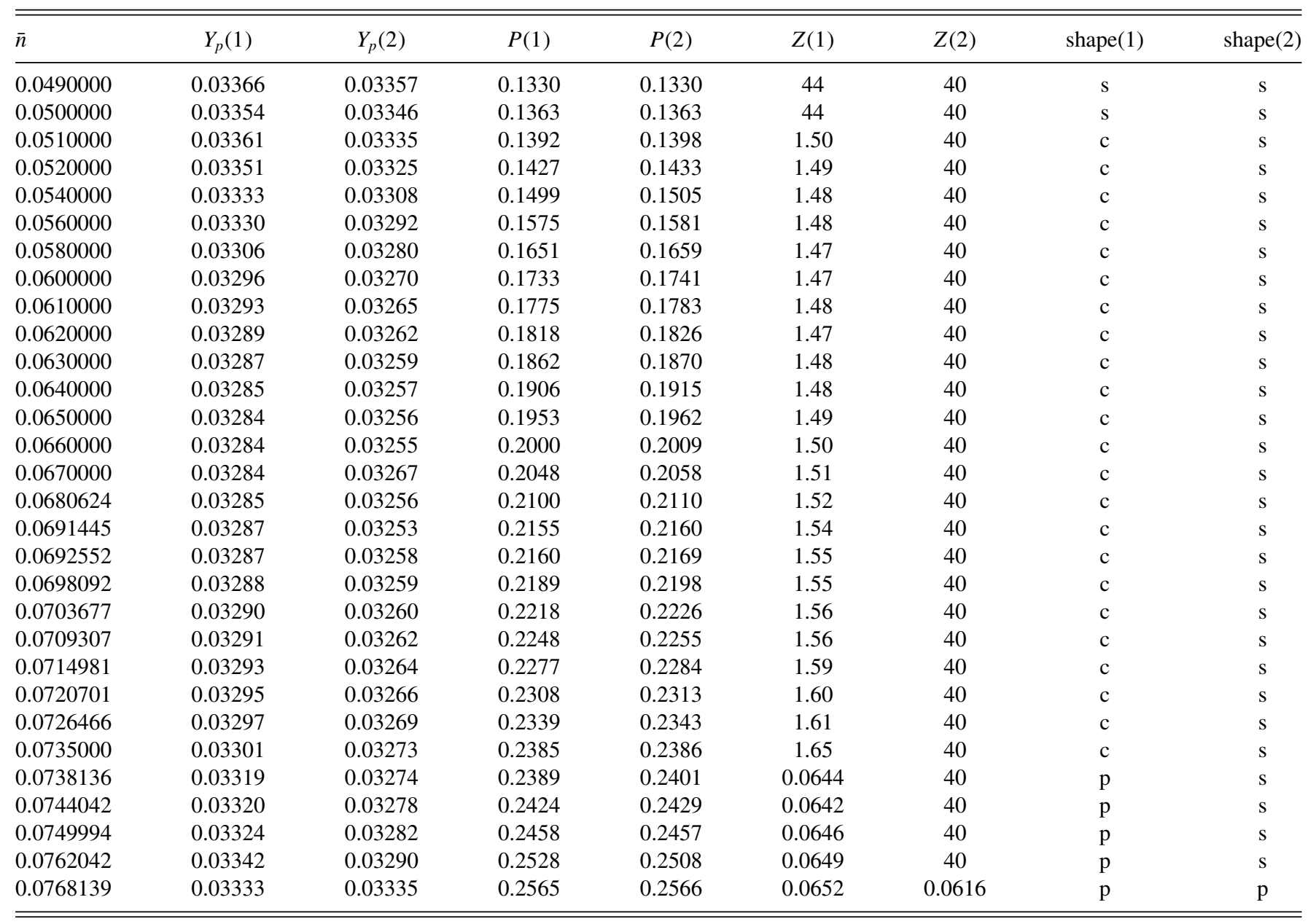

amount to $0.5 \%$, or less, of the total energy per nucleon, which is small enough to ensure the validity of the firstorder perturbation theory implicit in the application of the SI theorem.

Since the SI corrections in pasta are smoothly varying, there being no shell fluctuations, it follows that in spaghetti (and in lasagna) they must be compensating for the deviation of the ETF method from the smoothed HF method. This deviation, we recall, is the resultant of two opposed contributions: the inherent tendency of the ETF method to overbind compared to the smoothed HF method, and the inevitable underbinding that results from our parametrization of the density distributions. Thus it would appear that the former effect is dominant in cylinders and the latter in plates.

Concerning the final transition from plates to the homogeneous core, it was found in Ref. [2] that this transition occurs at $\bar{n}=0.0807555 \mathrm{fm}^{-3}$ for the BSk24 functional, but we cannot obtain reliable platelike solutions for higher densities than those shown in Table I. There are indications of a transition to homogeneous solutions already at $\bar{n}=0.07774283 \mathrm{fm}^{-3}$, but the convergence of these solutions is of dubious reliability. In any case, the calculations of the transition density in Ref. [2], being based on the assumption of small sinusoidal density fluctuations within the TF framework, do not necessarily yield an exact result.

Our results for cell shapes should be compared with those of Gögelein and Müther [21], who performed systematic inner-crust calculations using both $\mathrm{TF}$ and full $\mathrm{HF}+\mathrm{BCS}$ methods. Their results with the Skyrme functional SLy4 are completely different from ours: replacing the pure TF method with the $\mathrm{HF}+\mathrm{BCS}$ method actually leads to an increased presence of spaghetti, with the density of transition between spheres and cylinders being lowered from $0.066 \mathrm{fm}^{-3}$ to $0.042 \mathrm{fm}^{-3}$. On the other hand there is qualitative agreement with us when they repeat their calculations in the framework of the relativistic mean-field (RMF) method: Replacing the TF method with the RMF method leads to a reduced presence of spaghetti with the density of transition from spheres to cylinders being raised from $0.062 \mathrm{fm}^{-3}$ to $0.070 \mathrm{fm}^{-3}$. However, they do not find plates at all in their RMF calculations.

That the RMF and Skyrme calculations of Ref. [21] should have such radically different outcomes suggests that the presence of the pasta phases depends on the choice of functional. In this connection we note that our BSk24 functional used here has been fitted with precision to a large amount of nuclear data and subjected to a number of theoretical constraints of 

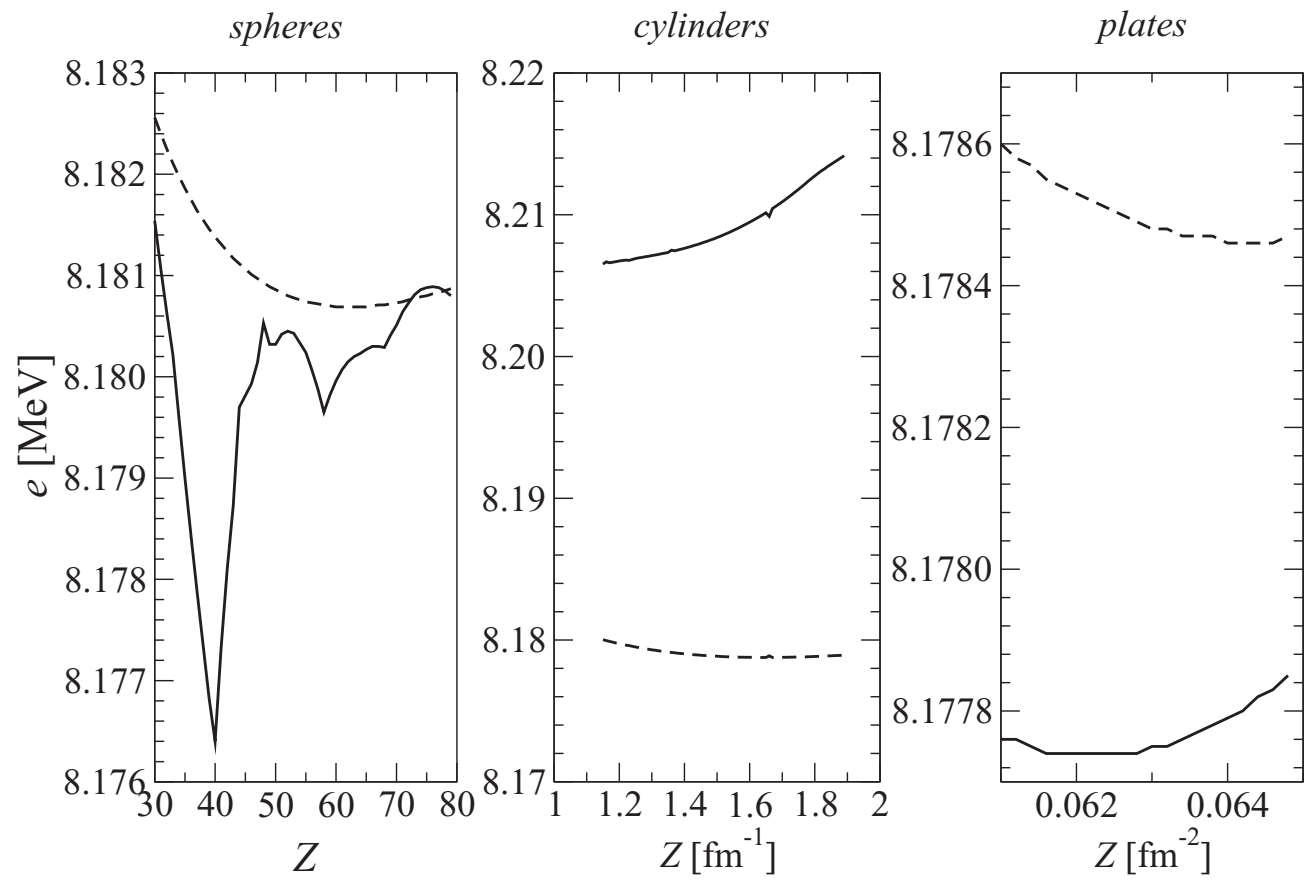

FIG. 2. Energy per nucleon at $\bar{n}=0.0738136 \mathrm{fm}^{-3}$ for spherical cells (left), spaghetti (center), and lasagna (right) as a function of proton number. Dotted curves: ETF, solid curves: ETFSI.

particular relevance to all regions of neutron stars. Moreover, the unified EoS based on this functional has also been found to be consistent with the analysis of the gravitational-wave signal GW170817 and of its electromagnetic counterparts [22]. Indeed, the results of Ref. [21] might simply reflect the existence of spurious shell effects arising from the imposition of periodic boundary conditions instead of Bloch boundary conditions, particularly in the case of free nucleons; these effects are expected to become increasingly important as the cell radius decreases with increasing density [23-25].

\section{B. Equation of state and composition}

Table II shows the pressure $P$, the proton fraction $Y_{p}$ and the value of $Z$ for the optimum equilibrium shapes shown in Table I. There are two columns for each of these three quantities, the one labeled "(1)" being for the pure ETF calculations, and the one labeled "(2)" for the calculations including the microscopic corrections (SI and pairing). Comparison of the two columns shows that the inclusion of the microscopic corrections has had very little impact on $P$ and $Y_{p}$, the shift always being less than $2 \%$ in the case of $Y_{p}$, and considerably smaller for $P$, even though the corresponding cell shape might be different. The deviation between the two values of $Z$ is somewhat larger, in the few cases where a meaningful comparison is possible (when the equilibrium cell shapes are not the same even the dimensions are different). We conclude that the microscopic corrections have a negligible impact on the EoS and the proton fraction. Thus the results of Refs. [2,10] for the EoS and the proton fraction, along with the fitted interpolation formulas, remain valid.

\section{CONCLUSIONS}

Our earlier ETF calculations [10] of pasta phases in the inner crust of neutron stars, performed with functional BSk24, have been repeated here with SI and pairing corrections, the latter made in the local-density approximation. We find that these microscopic corrections vary smoothly with respect to changing proton number, with no evidence for fluctuations, implying that there are no true shell effects in pasta, although there are strong effects in spherical WS cells arising from the SI corrections. (The absence of shell fluctuations that we find in pasta is to be contrasted with the conclusions of Ref. [13], which, using a more empirical method, found small, but not completely negligible, fluctuations.) On the other hand, the microscopic corrections are highly dependent on the cell shape, being positive for cylinders and negative for spheres or plates (over the density range for which pasta is found).

As a result of these microscopic corrections the spaghetti phase is completely eliminated and the lasagna phase confined to a thin layer close to the interface with the homogeneous core. This could be expected to lead to the elastic [26] and thermal properties [27] of neutron-star crusts being substantially different from those predicted in semiclassical and purely liquid-drop calculations. There will consequently be a significant impact on such neutron-star observables as continuous gravitational-wave emission [28], neutron-star oscillations [29] and their damping [30], the spin period of x-ray pulsars [31], and neutron-star cooling [32,33].

We stress that all the calculations reported here were performed for zero temperature. Finite-temperature effects always tend to reduce the quantal corrections, but at the temperatures $(T<0.1 \mathrm{MeV})$ prevailing in observed neutron 
stars, the zero-temperature calculations presented here should be valid [5]. On the other hand, if "freeze-out" in a cooling neutron star were to occur at significantly higher temperatures, then there could be a larger presence of pasta in the crust than we have indicated here.

A similar element of doubt in our conclusions might be introduced if one were to make an uncertainty analysis on the lines of that made by Thi et al. [34] in the framework of the compressible liquid-drop model. Conceivably, one might then find that it would no longer be possible to categorically exclude spaghetti, but our results presented here at least show that an extensive presence of pasta cannot be robustly assured within an ETFSI framework.

Given the sensitivity of our conclusions to a delicate balance between counteracting factors it would be interesting for the present calculations to be repeated with other functionals. Obvious candidates include the other Brussels-Montreal functionals studied in Ref. [2]. In any case, the functional BSk24 used here is preferred to the other functionals studied in Ref. [2] on account of a higher degree of reality and slightly better atomic-mass fits. More importantly, this functional has been found to be consistent with the neutron-star mass and radius constraints inferred from the gravitational-wave event GW170817 by the LIGO-Virgo collaboration as well as from the x-ray observations of millisecond pulsars by the NICER instrument [22] (see also Fig. 3 of Ref. [34]). However, the more recently developed functional BSk31 [35] is now preferred to BSk24 on account of its more realistic pairing properties, much better adapted to handling superfluidity in neutron stars, and we are currently repeating all our earlier calculations, including those of the present paper, with this new functional.

Bigger differences with respect to the present paper might be expected with functionals based on finite-range Gognytype effective interactions. Recently a unified EoS using such functionals has been constructed by Mondal et al. [36,37], with quantal corrections calculated by the SI method, but with the inner-crust calculations limited to spherical WS cells. There is a clear interest in generalizing these calculations to include pasta.

Finally, in asserting that one cannot reliably calculate cell shapes through purely liquid-drop or semiclassical approaches, one must stress that it is not sufficient simply to add shell fluctuations to such approaches. Rather, one must admit the possibility that the density distributions associated with liquid-drop or semiclassical calculations differ significantly from any reasonable smoothed approximation to the HF density distributions, and that shape-dependent energy shifts could consequently arise. On the other hand, we have shown that the microscopic corrections have a negligible impact on the EoS in the inner crust, i.e., on the pressure as a function of density, and on the proton fraction $Y_{p}$, as already noted in, for example, Ref. [3]. This conclusion holds even if the microscopic corrections lead to a change in the equilibrium cell shape. It is hence most unlikely that refinements to the calculation of the microscopic corrections could change significantly our predictions for the EoS and the proton fraction. Thus the results of Refs. [2,10] for the EoS and the proton fraction, along with the fitted interpolation formulas, remain valid, giving us a unified treatment based on the same functional BSk24 in all parts of the neutron star.

\section{ACKNOWLEDGMENTS}

Chen Wen Chao is thanked for his excellent management of the computing services of the Groupe de la Physique des Particules at the Université de Montréal. We thank A. Y. Potekhin for his critical comments on and continuing interest in this work. This work was supported by the Fonds de la Recherche Scientifique (Belgium) under Grants No. PDR T.004320 and No. IISN 4.4502.19. J.M.P. thanks the Université Libre de Bruxelles for the award of a "Chaire internationale" during the tenure of which the present work was initiated. This work was also partially supported by the European Cooperation in Science and Technology Action CA16214.

\section{APPENDIX: COULOMB FIELD IN GLOBALLY NEUTRAL WIGNER-SEITZ CELLS}

The Coulomb field $U_{C}$ in the WS cell can be found from the solution of Poisson's equation in the WS cell,

$$
\nabla^{2} U_{C}(\boldsymbol{r})=-4 \pi e^{2} n_{\mathrm{ch}}(\boldsymbol{r}) .
$$

Recalling that the cell is electrically charge neutral, we have

$$
\int n_{\mathrm{ch}}(\boldsymbol{r}) d^{3} \boldsymbol{r}=0,
$$

There are three cases of interest for the present paper.

\section{Spheres}

In this case Eq. (A1) becomes

$$
\frac{1}{r^{2}} \frac{d}{d r}\left[r^{2} \frac{d U_{C}(r)}{d r}\right]=-4 \pi e^{2} n_{\mathrm{ch}}(r) .
$$

Integrating once yields

$$
\frac{d U_{C}(r)}{d r}=-\frac{4 \pi e^{2}}{r^{2}} u(r),
$$

where

$$
u(r)=\int_{0}^{r} n_{\mathrm{ch}}\left(r^{\prime}\right) r^{\prime 2} d r^{\prime}
$$

and we have made use of the requirement that $d U_{C} / d r$ must vanish at the origin. A second integration leads to

$$
U_{C}(r)=4 \pi e^{2} \int_{r}^{R} \frac{u\left(r^{\prime}\right)}{r^{\prime 2}} d r^{\prime},
$$

where we have set $U_{C}(R)=0$, as appropriate on the surface of a spherically symmetric charge distribution that is globally neutral. Integrating this last expression by parts and using the result

$$
u(R)=0,
$$

which follows from Eq. (A5) because of the neutrality condition (A2), we find

$$
U_{C}(r)=4 \pi e^{2} \int_{r}^{R} n_{\mathrm{ch}}\left(r^{\prime}\right)\left(r^{\prime}-r^{\prime 2} / r\right) d r^{\prime} .
$$


We have used this result extensively in the past $[2,4-7,10]$ without presenting it explicitly.

\section{Cylinders}

In this case, Poisson's equation reads

$$
\frac{1}{\eta} \frac{d}{d \eta}\left[\eta \frac{d U_{C}(\eta)}{d \eta}\right]=-4 \pi e^{2} n_{\mathrm{ch}}(\eta)
$$

Integrating once yields

$$
\frac{d U_{C}(\eta)}{d \eta}=-\frac{4 \pi e^{2}}{\eta} u(\eta)
$$

where

$$
u(\eta)=\int_{0}^{\eta} n_{\mathrm{ch}}\left(\eta^{\prime}\right) \eta^{\prime} d \eta^{\prime}
$$

A second integration leads to

$$
U_{C}(\eta)=4 \pi e^{2} \int_{\eta}^{R} \frac{u\left(\eta^{\prime}\right)}{\eta^{\prime}} d \eta^{\prime},
$$

setting $U_{C}(R)=0$. Integrating by parts we obtain

$$
U_{C}(\eta)=-4 \pi e^{2}\left[\ln (\eta) u(\eta)+\int_{\eta}^{R} n_{\mathrm{ch}}\left(\eta^{\prime}\right) \ln \left(\eta^{\prime}\right) \eta^{\prime} d \eta^{\prime}\right] .
$$

Equation (24) follows after remarking that Eq. (A7) holds again because of the neutrality condition (A2).

\section{Plates}

Poisson's equation reads

$$
\frac{d}{d z}\left[\frac{d U_{C}(z)}{d z}\right]=-4 \pi e^{2} n_{\mathrm{ch}}(z)
$$

whence

$$
\frac{d U_{C}(z)}{d z}=-4 \pi e^{2} u(z)
$$

with

$$
u(z)=\int_{0}^{z} n_{\mathrm{ch}}\left(z^{\prime}\right) d z^{\prime} .
$$

Since Eq. (A7) holds again, because of global neutrality, we can integrate Eq. (A15) by parts to find Eq. (33).
[1] D. Blaschke and N. Chamel, in The Physics and Astrophysics of Neutron Stars, Astrophysics and Space Science Library, Vol. 457, edited by L. Rezzolla, P. Pizzochero, D. I. Jones, N. Rea, and I. Vidaña (Springer, Berlin, 2018), pp. 337-400.

[2] J. M. Pearson, N. Chamel, A. Y. Potekhin, A. F. Fantina, C. Ducoin, A. K. Dutta, and S. Goriely, Mon. Not. Roy. Astron. Soc. 481, 2994 (2018); 486, 768 (2019).

[3] B. K. Sharma, M. Centelles, X. Viñas, M. Baldo, and G. F. Burgio, Astron. Astrophys. 584, A103 (2015).

[4] A. K. Dutta, M. Onsi, and J. M. Pearson, Phys. Rev. C 69, 052801(R) (2004).

[5] M. Onsi, A. K. Dutta, H. Chatri, S. Goriely, N. Chamel, and J. M. Pearson, Phys. Rev. C 77, 065805 (2008).

[6] J. M. Pearson, N. Chamel, S. Goriely, and C. Ducoin, Phys. Rev. C 85, 065803 (2012).

[7] J. M. Pearson, N. Chamel, A. Pastore, and S. Goriely, Phys. Rev. C 91, 018801 (2015).

[8] M. Bender, P.-H. Heenen, and P.-G. Reinhard, Rev. Mod. Phys. 75, 121 (2003).

[9] M. Shelley and A. Pastore, Universe 6, 206 (2020).

[10] J. M. Pearson, N. Chamel, and A. Y. Potekhin, Phys. Rev. C 101, 015802 (2020).

[11] S. Goriely, N. Chamel, and J. M. Pearson, Phys. Rev. C 88, 024308 (2013).

[12] N. Chamel, S. Goriely, and J. M. Pearson, Phys. Rev. C 80, 065804 (2009).

[13] K. Oyamatsu and M. Yamada, Nucl. Phys. A 578, 181 (1994).

[14] M. Brack, C. Guet, and H.-B. Håkansson, Phys. Rep. 123, 275 (1985).
[15] S. Goriely, N. Chamel, and J. M. Pearson, Eur. Phys. J. A 42, 547 (2009).

[16] N. Chamel and S. Goriely, Phys. Rev. C 82, 045804 (2010).

[17] J. W. Negele and D. Vautherin, Nucl. Phys. A 207, 298 (1973).

[18] M. Baldo, E. E. Saperstein, and S. V. Tolokonnikov, Nucl. Phys. A 775, 235 (2006).

[19] See Supplemental Material at http://link.aps.org/supplemental/ 10.1103/PhysRevC.105.015803 for full numerical results.

[20] M. Hashimoto, H. Seki, and M. Yamada, Prog. Theor. Phys. 71, 320 (1984).

[21] P. Gögelein and H. Müther, Phys. Rev. C 76, 024312 (2007).

[22] L. Pérot, N. Chamel, and A. Sourie, Phys. Rev. C 100, 035801 (2019) .

[23] N. Chamel, S. Naimi, E. Khan, and J. Margueron, Phys. Rev. C 75, 055806 (2007).

[24] W. G. Newton and J. R. Stone, Phys. Rev. C 79, 055801 (2009).

[25] B. Schuetrumpf, W. Nazarewicz, and P.-G. Reinhard, Phys. Rev. C 93, 054304 (2016).

[26] A. Y. Potekhin and C. J. Pethick, Phys. Lett. B 427, 7 (1998).

[27] D. Durel and M. Urban, Phys. Rev. C 97, 065805 (2018); 102, 029901(E) (2020).

[28] M. E. Caplan, A. S. Schneider, and C. J. Horowitz, Phys. Rev. Lett. 121, 132701 (2018).

[29] H. Sotani, Mon. Not. Roy. Astron. Soc. Lett. 417, L70 (2011).

[30] D. G. Yakovlev, M. E. Gusakov, and P. Haensel, Mon. Not. Roy. Astron. Soc. 481, 4924 (2018).

[31] J. Pons, D. Viganò, and N. Rea, Nat. Phys. 9, 431 (2013). 
[32] M. E. Gusakov, D. G. Yakovlev, P. Haensel, and O. Y. Gnedin, Astron. Astrophys. 421, 1143 (2004).

[33] Zidu Lin, M. E. Caplan, C. J. Horowitz, and C. Lunardini, Phys. Rev. C 102, 045801 (2020).

[34] H. Dinh Thi, T. Carreau, A. F. Fantina, and F. Gulminelli, Astron. Astrophys. 654, A114 (2021).
[35] S. Goriely, N. Chamel, and J. M. Pearson, Phys. Rev. C 93, 034337 (2016).

[36] C. Mondal, X. Viñas, M. Centelles, and J. N. De, Phys. Rev. C 102, 015802 (2020).

[37] X. Viñas, C. Gonzales-Boquera, M. Centelles, C. Mondal, and L. Robledo, Symmetry 13, 1613 (2021). 